\title{
Mathematical modeling of the nonstationary sugar mass crystallization process in the vacuum machine heating tube wall layer
}

\author{
Taras Pohorilyi, Valerii Myronchuk
}

1. Technological equipment and computer design technology, National University of Food Technologies,

UKRAINE, Kiev, Volodymyrska Street 68, E-mail: pogorilyytm@ukr.net

\begin{abstract}
A mathematical modeling of the nonstationary sugar mass crystallization process from the unsteady heat and mass transfer process viewpoint are considered. Sugar massecuite as a cellular model represented. With the simultaneous unsteady heat and diffusion mass transfer problems solution between the system cells were considered variable thermal physics characteristics.
\end{abstract}

Keywords - mass sucrose crystallization, unstable heat and diffusion mass transfer.

\section{Introduction}

The process of mass crystallization of sucrose in the sugar massecuite boiling is the most energy intensive industrial production of sugar. For its control and reduce the energy necessary to create a mathematical model of the process that would fully describe it. It is this and devoted to this work.

\section{Materials and methods}

Taking into account all thermophysical, technological and hydrodynamic characteristics when creating a mathematical model of mass crystallization of sucrose is extremely difficult and practically impossible. As a result, a number of simplifications were made. Thus, the mathematical model of the mass crystallization process of sucrose is idealized.

All sucrose mass crystallization processes are considered from the point of view of the non-stationary variable-time heat and mass transfer process. Sugar massecuite was considered in cellular models terms: crystal sugar, which is surrounded by a corresponding volume crystal layer between sucrose solution. Find the simultaneous non-stationary heat transfer problems solution for each cell separately and non-stationary diffusion mass transfer problems between the corresponding intercrystal sucrose solution cells by analytical methods is extremely difficult. In connection with this, at the same time, non-stationary heat conduction problems and nonstationary diffusion mass transfer problems for the intercrystalline sucrose solutions areas by numerical methods were solved.

\section{Results}

On the basis of the simultaneous system solution of seven non-stationary heat conduction problems and three separate non-stationary diffusion mass transfer problems, a non-stationary temperature distributions for the entire cells system each component were found. Concentration distributions in the corresponding intercrystal sucrose solution regions were also found for stable and variables of the thermophysical characteristics of the cells of the system.

\section{Conclusion}

The received number of different non-stationary temperatures and concentrations distribution in the cells system allows us to determine the more optimal conditions for boiling sugar massecuite a with minimal energy consumption and minimal losses of the finished product. 